\title{
Real-time AGC dispatch units considering wind power and ramping capacity of thermal units
}

\author{
Jingyi ZHANG, Chao LU, Jie SONG (1) (ه), \\ Junbo ZHANG
}

\begin{abstract}
The high penetration of wind energy sources in power systems has substantially increased the demand for faster-ramping thermal units participating in the frequency regulation service. To fulfill the automatic generation control (AGC) and compensate the influence of wind power fluctuations simultaneously, ramping capacity should be considered in the dispatch model of thermals. Meanwhile, conventional methods in this area do not take the impact of transmission loss into the dispatch model, or rely on offline network model and parameters, failing to reflect the real relationships between the wind farms and thermal generators. This paper proposes an online approach for AGC dispatch units considering the above issues. Firstly, the power loss sensitivity is online identified using recursive least square method based on the real-time data of phasor measurement units. It sets up power balance constraint and results in a more accurate dispatch model. Then, an improved multi-objective optimization model of dispatch is proposed and a connection is established between the thermal units with fast ramping capacity and the wind farms with rapid fluctuations. Genetic algorithm is used to solve the dispatch model. The proposed method is compared with conventional methods in simulation case in the IEEE 30-bus system. Finally, simulation results verify the validity and the feasibility of identification method and optimization model.
\end{abstract}

CrossCheck date: 25 February 2015

Received: 31 August 2014/ Accepted: 25 February 2015/Published online: 26 July 2015

(C) The Author(s) 2015. This article is published with open access at Springerlink.com

J. ZHANG, C. LU, J. ZHANG, State Key Laboratory of Power

System, Department of Electrical Engineering, Tsinghua

University, Beijing 100084, China

J. SONG, Department of Industrial Engineering and

Management, Peking University, Beijing, China

$(\bowtie)$ e-mail: songjie@coe.pku.edu.cn
Keywords Ramping capacity, Loss sensitivity, Automatic generation control (AGC) optimal dispatch, On-line identification, Genetic algorithm

\section{Introduction}

With the increase of wind energy penetration and power consumption, the dispatch of automatic generation control (AGC) units becomes an important issue in the field of frequency control [1]. In recent years, wind energy, as a kind of clean and abundant energy, occupies an increasingly important position. The random characteristic of wind speed results in a serious degree of disturbance in the power system, leading to the increasing need of faster-ramping thermal units. Lots of work have been proposed to improve the ramping capacity of thermal units [2,3]. Reference [2] improved the ramping capacity of thermal units by cooperating circulating fluid bed (CFB) units with heating units. Reference [3] achieved its goal by optimizing primary air pressure controlling loop, adding the loop of caloricity calibration to adapt to the coal quality variation, and designing condensate throttling and parameter adaptive method of key parameters in boiler and turbine.

The AGC units dispatch is essentially an optimization problem, therefore, different optimization methods have been used to solve this issue, such as dynamic programming method [4], ant colony optimization, genetic algorithm (GA) [5, 6], particle swarm optimization (PSO) technique [7-9], and improved immune algorithm [10], etc. Despite different optimization methods, the objective function limits to the minimization of generation cost without considering the ramping capacity. References [2,3] didn't consider both objectives in the same framework, which leads to application difficulties.

On the other hand, frequency control requires the real-time balance of generation, load and loss, and meets the generation limits. In many existing studies, the transmission loss is 
ignored, therefore, the result of dispatching is suboptimal. This is because the transmission loss affects the output of generation units directly, thus affecting the power distribution. Conventional loss calculation is based on B-coefficient method [11], and then is improved with inverse Jacobian transpose method [12] and Z-bus loss allocation [13]. These methods are based on the off-line models and parameters which are contained with bias in real power systems, therefore, the performances are doubtful. With the implementation of phasor measurement units (PMUs), the active power of most nodes can be real-time measured, which provides a new direction to calculate the transmission loss.

To solve the above issues, a real-time AGC dispatch units approach considering wind power and ramping capacity of thermal units is proposed in this paper. Firstly, a transmission loss sensitivity is identified and the transmission loss is calculated. Then, a multi-objective optimization model combining with generation cost (considering the transmission loss) and fast-ramping capacity is proposed.

The remainder of this paper is organized as follows: Section 2 proposes the recursive least square (RLS) method to identify the loss sensitivity matrix online. Section 3 presents a multi-objective optimization model of AGC dispatch units. Section 4 uses the GA to solve the optimal allocation problem, and the simulation result of IEEE 30-bus case indicates the validity of the dispatch model and the methodology of loss sensitivity matrix online identification. Section 5 draws conclusion.

\section{Online identification of loss sensitivity}

During the processes of AGC optimal allocation, the optimization is subject to the following power balance constraint.

$\sum_{i \in N D} P_{d i}+P_{l}=\sum_{j \in N G} P_{G j}+\sum_{l \in N W} P_{w l}$

where $N D$ is the set of load nodes; $N G$ is the set of thermal generation units; $N W$ is the set of wind generation units; $P_{d i}$ is the demand of Node $i ; P_{l}$ is the system transmission loss; Their sum is the effective load to be satisfied; $P_{G j}$ is the generation of unit $j$ in thermal power plants; $P_{w l}$ is the generation of unit $l$ in wind power plants.

How to accurately and rapidly identify the transmission losses of current network during the process of AGC optimal allocation is of importance in practice. The development of PMUs and wide area measurement system (WAMS) makes it possible to achieve the real-time measurement of state variables in the power system. In the platform of WAMS, the variation of all the units can be obtained directly, thus, the transmission loss can also be calculated using the real-time measurement data. The constraint of power balance can also be described with loss sensitivity, which is on-line identified with the data of current operating status.

$$
\begin{aligned}
& \sum_{i \in N D} \Delta P_{d i}+\sum_{i \in N D} \frac{\partial P_{l}}{\partial P_{d i}} \Delta P_{d i}+\sum_{j \in N G} \frac{\partial P_{l}}{\partial P_{G j}} \Delta P_{G j}+\sum_{l \in N W} \frac{\partial P_{l}}{\partial P_{w l}} \Delta P_{w l} \\
& =\sum_{j \in N G} \Delta P_{G j}+\sum_{l \in N W} \Delta P_{w l}
\end{aligned}
$$

where $\Delta P_{d i}$ is the change of the demand at Node $i ; \Delta P_{G j}$ is the change of the generation of thermal unit $j ; \Delta P_{w l}$ is the change of the generation of wind unit $l ; \partial P_{l} / \partial P_{d i}$ is the loss sensitivity to the active power at load Node $i ; \partial P_{l} / \partial P_{G j}$ is the loss sensitivity to the active power at thermal unit $j ; \partial P_{l} / \partial P_{w l}$ is the loss sensitivity to the active power at wind unit $l$.

Transmission loss is related to the changes of power flows. The change of active power output at Node $k\left(\Delta P_{k}\right)$ will result in the change of transmission loss $\left(\Delta P_{l}\right)$ with the active power output of other nodes (except for the balance node) unchanged. Thus, under current system operating status, the relation between the active power output of Node $k$ and transmission loss can be approximately described as follows:

$\Delta P_{l}=\frac{\partial P_{l}}{\partial P_{k}} \Delta P_{k}+\varepsilon_{k}$

where the partial derivative $\partial P_{l} / \partial P_{k}$ is the loss sensitivity to the active power at Node $k ; \varepsilon_{k}$ is the deviation that the approximate description above brings.

Similarly, the number of the nodes (except for the balance node) at which the active power change extends to $N$, then the approximate relation between the power output of $N$ nodes and transmission losses can be described as:

$$
\begin{aligned}
\Delta P_{l}= & \frac{\partial P_{l}}{\partial P_{1}} \Delta P_{1}+\frac{\partial P_{l}}{\partial P_{2}} \Delta P_{2}+\cdots+\frac{\partial P_{l}}{\partial P_{k}} \Delta P_{k}+\cdots \\
& +\frac{\partial P_{l}}{\partial P_{N}} \Delta P_{N}+\varepsilon
\end{aligned}
$$

where $\varepsilon$ is the deviation of all.

For actual power system, in $m$ continuous sampling intervals, Equation (4) can be expanded as

$$
\begin{aligned}
& {\left[\begin{array}{c}
\Delta P_{l}^{(1)} \\
\Delta P_{l}^{(2)} \\
\vdots \\
\Delta P_{l}^{(m)}
\end{array}\right]=} {\left[\begin{array}{cccc}
\Delta P_{1}^{(1)} & \Delta P_{2}^{(1)} & \cdots & \Delta P_{N}^{(1)} \\
\Delta P_{1}^{(2)} & \Delta P_{2}^{(2)} & \cdots & \Delta P_{N}^{(2)} \\
\vdots & \vdots & \ddots & \vdots \\
\Delta P_{1}^{(m)} & \Delta P_{2}^{(m)} & \cdots & \Delta P_{N}^{(m)}
\end{array}\right]\left[\begin{array}{c}
\frac{\partial P_{l}}{\partial P_{1}} \\
\frac{\partial P_{l}}{\partial P_{2}} \\
\vdots \\
\frac{\partial P_{l}}{\partial P_{N}}
\end{array}\right] } \\
&+\left[\begin{array}{c}
\varepsilon^{(1)} \\
\varepsilon^{(2)} \\
\vdots \\
\varepsilon^{(m)}
\end{array}\right]
\end{aligned}
$$


The matrix form is described as:

$\Delta \boldsymbol{P}_{l}=\Delta \boldsymbol{P} \frac{\partial \boldsymbol{P}_{l}}{\partial \boldsymbol{P}}+\varepsilon$

where $\Delta \boldsymbol{P}$ is the matrix of the changes of active power of different nodes; $\partial \boldsymbol{P}_{l} / \partial \boldsymbol{P}$ is the vector of the loss sensitivity; $\varepsilon$ is the vector of deviation.

The matrix $\boldsymbol{\Delta P}$ in (6) can be measured by WAMS, while the vector $\Delta \boldsymbol{P}_{l}$ can be calculated through the data of realtime measurement. At the condition of $m=N$, the unique solution can be obtained when $\Delta \boldsymbol{P}$ is invertible. If $\Delta \boldsymbol{P}$ is not invertible, the sampling times $m$ can be increased to make $\Delta \boldsymbol{P}$ column full rank. As for the condition of $m>N$, the vector of loss sensitivity can be obtained with least square (LS) method. For the character that RLS algorithm does not need to reserve all the data, (once a new set of data arrives, the parameters will be estimated), the required amount of calculation and storage space is very small. Therefore, online real-time identification can be achievable. From the mathematical point of view, (4) is expressed as a standard multiple linear regression equation, where the active power changes of different units' output are the independent variables, the transmission loss is the dependent variable, and the sensitivity coefficients are the regression coefficients. Equation (6) is established by repeatedly creating (4) at different continuous sampling intervals, furthermore, the sensitivity coefficients will be calculated using. the modeling method of multiple regression. The results of loss sensitivity identification are used for the AGC dispatch. Therefore, the frequency of parameter estimation is designed to be consistent with the AGC command signal.

\section{Optimization model considering ramping capacity of thermal units}

\subsection{Objective function}

\subsubsection{Economical efficiency}

The goal of economic dispatch is to minimize the total cost of power generation by reasonably distributing the active power output of generator sets under the premise of generating and operating constraints.

The phenomenon when the turbine intake valve turns wire drawing superimposes a pulsating effect on the unit consumption curve, resulting in a valve-point effect [14]. In addition to the cost of generation fuel in the turbine, the energy cost produced by steam turbine valve point is included in the overall power cost in this paper. The economic optimization goal expression without considering wind power operation cost is described as: $\min f\left(P_{G j}\right)=\sum_{j \in N G} F_{j}\left(P_{G j}\right)+E_{j}\left(P_{G j}\right)$

where $f\left(P_{G j}\right)$ is the total cost of the generation; $F_{j}\left(P_{G j}\right)$ is the cost of generation fuel of thermal unit $j ; E_{j}\left(P_{G j}\right)$ is the energy cost produced by turbine's valve-point of thermal unit $j$.

$F_{j}\left(P_{G j}\right)=a_{j}\left(P_{G j}\right)^{2}+b_{j} P_{G j}+c_{j}$

$E_{j}\left(P_{G j}\right)=\left|e_{j} \sin \left[f_{j}\left(P_{G j \min }-P_{G j}\right)\right]\right|$

where $a_{j}, b_{j}, c_{j}$ are the fuel cost coefficients; $P_{G j \min }$ is the minimum output of thermal unit $j ; e_{j}$ and $f_{j}$ are the coefficients of valve point effects.

\subsubsection{Economical efficiency}

The emergence of high renewable energy source penetration such as wind power in the energy mix of power systems has substantially increased the need for fasterramping resources participating in the frequency regulation. Meanwhile, with the development of fast-ramping technology, there is a gap of ramping capacity between different thermal units. Under such circumstance, how to allocate the output of thermal units to make the regulation time of AGC shortest by allocating more output to the units with faster-ramping capacity is the goal of rapidity.

The regulation time of thermal unit $j$ can be described as follows:

$t_{j}=\left|P_{G j}-P_{G j n o w}\right| / V_{j}=\left|\Delta P_{G j}\right| / V_{j}$

where $V_{j}$ is the regulation rate of thermal unit $j$.

When all the regulation units adjust with their outputs at the same time, the AGC regulation time of the area is the longest regulation time of all units.

The regulation time of AGC can be described as:

$T=\max _{j \in N G} t_{j}$

where $T$ is the regulation time of AGC.

In order to make the time of AGC regulation shortest, we need to make the time of the slowest unit shortest. The objective function of rapidity can be expressed as follows:

$\min T=\min \max _{j \in N G}\left|P_{G j}-P_{G j \text { now }}\right| / V_{j}$

\subsubsection{Cooptimization of economy and rapidity}

The objective functions of economy and rapidity have different dimensions, so we decide to sum them up after normalizing respectively, the co-optimization of them can be described as follows: 
$\min \left\{\alpha_{s} \frac{\max _{j \in N G} t_{j}-T_{o p i}}{T_{o p i}}+\alpha_{p} \frac{f\left(P_{G j}\right)-C_{o p i}}{C_{o p i}}\right\}$

where $\alpha_{s}, \alpha_{p}$ are the weight coefficients of rapidity and efficiency, and $\alpha_{s}+\alpha_{p}=1 ; T_{o p i}, C_{o p i}$ are constants, the purpose of which is to keep the normalized values of economy and rapidity in the same order of magnitude. In this paper, the value of $C_{o p i}$ to the optimal cost is assigned when only considering efficiency, and $T_{o p i}$ to the optimal time when only considering rapidity.

\subsection{Operating constraints}

1) The power balance constraint using loss sensitivity

As mentioned above, the real-time changes of units' outputs and transmission loss measured by PMUs are utilized to identify and update the loss sensitivity matrix in real time. We can obtain the transmission loss during the optimization process by the loss sensitivity matrix, and then the constraint of the power balance can also be described with the loss sensitivity:

$$
\begin{aligned}
& \sum_{i \in N D} \Delta P_{d i}+\sum_{i \in N D} \frac{\partial P_{l}}{\partial P_{d i}} \Delta P_{d i}+\sum_{j \in N G} \frac{\partial P_{l}}{\partial P_{G j}} \Delta P_{G j}+\sum_{l \in N W} \frac{\partial P_{l}}{\partial P_{w l}} \Delta P_{w l} \\
& =\sum_{j \in N G} \Delta P_{G j}+\sum_{l \in N W} \Delta P_{w l}
\end{aligned}
$$

It is worth mentioning that the calculation speed is an important index of power system dispatch. Compared with the loss sensitivity calculation method based on the trend of network, the methodology in this paper can enhance the calculation speed with the assurance of the computing accuracy at the same time, which can meet the requirement of real-time dispatch better.

2) Capacity constraints of thermal generators

The output of thermal generators has operating range, while the constraint can be described as follows:

$P_{G j \max } \geq P_{G j} \geq P_{G j \min }$

3) The capacity constraints of spinning reserve

To guarantee its safety and reliability, the system needs to preserve sufficient reserve capacity, spinning reserve in especial. The capacity of spinning reserve is used to reduce the effect of errors in load prediction when there is no wind farm in the network. With the penetration of wind farm, the effects of errors caused by wind power prediction should be taken into account. Under this circumstance, two different types of spinning reserve should be considered, i.e., upward spinning reserve and downward spinning reserve. The upward spinning reserve is used to deal with sudden increase of load and unexpected drop of wind power, while the downward spinning reserve is used to cope with the opposite situation. When the downward spinning reserve is not considered in dispatch of the system, it may result in frequent start-stop operations of thermal generating units [14].

The constraint of upward spinning reserve can be described as:

$$
\begin{aligned}
& \sum_{j \in N G} S_{u s}^{j} \geq P_{d} L+P_{w} w_{u} \\
& S_{u s}^{j}=\min \left(S_{u s \max }^{j}, P_{G j \max }-P_{G j}\right)
\end{aligned}
$$

where $S_{u s}^{j}$ is the capacity of upward spinning reserve provided by generator unit at present; $L$ is the requirement of upward spinning reserve made by the prediction error of all the load in the system; $w_{u}$ is the requirement of upward spinning reserve made by the prediction error of wind power; $S_{u s \max }^{j}$ is the maximum of upward spinning reserve that thermal unit $j$ can offer.

The constraint of downward spinning reserve can be described as:

$\sum_{j \in N G} S_{d s}^{j} \geq\left(P_{w \max }-P_{w}\right) w_{d}$

$S_{d s}^{j}=\min \left(S_{d s \max }^{j}, P_{G j}-P_{G j \min }\right)$

where $S_{d s}^{j}$ is the capacity of downward spinning reserve provided by generator unit at present; $P_{w \max }$ is the maximum capacity of the wind farm; $w_{d}$ is the requirement of downward spinning reserve made by the prediction error of wind power; $S_{d s \max }^{j}$ is the maximum of downward spinning reserve that thermal unit $j$ can offer.

\section{Simulation on IEEE 30-bus system}

\subsection{Simulation system}

IEEE 30-bus system is used to explain the identification of loss sensitivity methodology and the validity of the optimal dispatch model is proved. The test case is shown in Fig. 1.

The data of the current active power supply, the upper and lower limits of active power supply, the coefficients of the cost and the ramping rates of thermal units are shown in Appendix Table 4. The corresponding number of each unit is provided according to its bus number. Unit 1 is the balancer of the system, which can regulate its power supply within upper and lower limits. Unit 8 stands for the wind farm with the specified active power supply of 0.3 p.u., which including 20 wind units based on the good performance of variable. The overall load of the system is 2.883 p.u. and the basic value of the power is 100 MVA [15]. The simulation platform is MATLAB 7.0. 


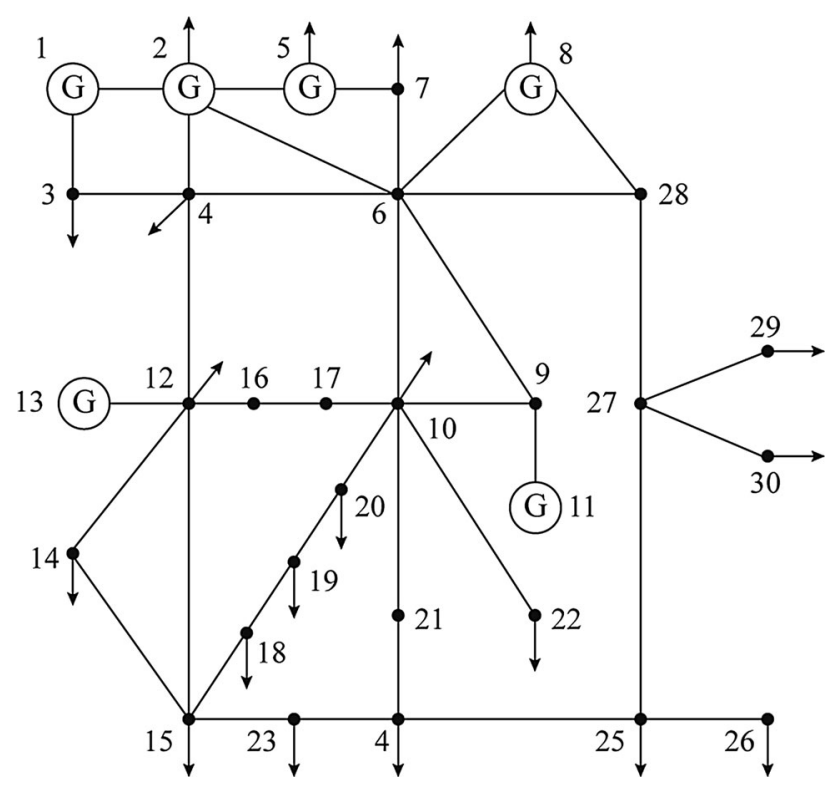

Fig. 1 IEEE 30-bus system

\subsection{Identification of loss sensitivity}

Under the present operating circumstance, we bring a stochastic perturbation with small amplitude into the active power outputs of all the units (except for the balancer) in the system to imitate the changes of the system (Apply $0.5 \%$ of current outputs for the thermal units as a stochastic interruption, while $2.5 \%$ of current outputs for the wind unit to simulate its sharper change). The loss sensitivities of units are identified in a real-time scale with the utilization of the active power supply of common units and variation of transmission losses supervised at different time, where RLS method is also used. To prove the feasibility and superiority of the loss sensitivity identification method clearly, the simulation result is compared with that of the B-coefficient method and the inverse Jacobian method as shown in Table 1. The loss sensitivity of the balancer in the system is set to 0 as a reference. The time consumptions of the three methods are also provided in the Table 1. A relative error is used to describe the similarity of the results between the proposed method and inverse Jacobian transpose method. Its definition formula is as follows:

$\delta_{R-J}^{i}=\left|\frac{S_{R}^{i}-S_{J}^{i}}{S_{J}^{i}}\right| \times 100 \%$

where $\delta_{R-J}^{i}$ is the relative error of loss sensitivity between the proposed method and inverse Jacobian transpose method at bus $i ; S_{R}^{i}$ and $S_{J}^{i}$ are the loss sensitivity results at bus $i$ calculated by the proposed method and inverse Jacobian transpose method, respectively. $\delta_{R-J}^{i}$ is also shown in Table 1.
From Table 1, we can obtain the similar results by the identification with the RLS method, B-coefficient method, and inverse Jacobian method, thus guaranteeing the validity of the method.

Compared the B-coefficient method with inverse Jacobian method, the method in this paper can improve the computing speed on the assurance of full use of the data, which is helpful to the real-time generating control. It is worth mentioning that the algorithm utilizes the current disturbance data to reflect the influence of the output of the generation units on the transmission losses. The proposed method utilizes the data that can reflect the real operational condition of power systems, while the B-coefficient method and the inverse Jacobian method utilize the off-line data and models.

Next, we will observe the effect of the perturbation amplitude on the sensitivity identification by applying different ranges of random disturbance on the nodes. We apply $0.2 \%$, $0.5 \%, 1 \%, 2 \%, 2.5 \%$ of current outputs for the thermal units, while $1 \%, 2.5 \%, 5 \%, 10 \%, 12.5 \%$ are used for the wind unit to simulate perturbation, obtaining five sensitivity identification results under different conditions, respectively. Then we calculate the root-mean square error between the identification result and the result of inverse Jacobian method, respectively, as shown in Fig. 2. From Fig. 2, we can see that the perturbation amplitude within a certain range does not have an obvious effect on the identification results. Furthermore, it can illustrate that the loss identification method has wide applicability under actual operation status with different disturbance amplitudes.

\subsection{Dispatch results of AGC units}

The power-balance constraint is set up with the loss sensitivity matrix calculated in Section 4.2 , and then the AGC dispatch of IEEE 30-bus system is modeled with taking ramping capacity into consideration. Genetic algorithm (GA) is used to solve the optimization model. Because the computing time increases correspondingly with the increase of genetic algebra, we hope to reduce genetic iterations as many as possible on the basis of accuracy. Thus, we make the assumption that the genetic iteration process will be complete if the variance of all species' objective values is less than the given tolerance to improve the genetic algorithm. The parameters of GA are selected through experiments. The population is 500; the maximum number of generation is 5000; the crossover probability is 0.7 .

When taking consideration of the output changes of wind power units, we suppose that the output of wind farm decreased to 0 because of the change of wind speed. The active power of the system to adjust is 0.3 p.u. With the AGC units dispatch model of IEEE-30, the effects of the weight coefficient to rapidity, the loss sensitivity, the location of faster-ramping unit on the result of optimization are analyzed as follows: 
Table 1 Results of loss sensitivity

\begin{tabular}{lllllllr}
\hline Method & Unit 1 & Unit 2 & Unit 5 & Unit 8 & Unit 11 & Unit 13 & Computing time (ms) \\
\hline RLS & 0 & -0.02749 & -0.06721 & -0.05031 & -0.05051 & -0.04073 & 12.566 \\
B-coefficient & 0 & -0.03013 & -0.06498 & -0.04879 & -0.04858 & -0.03765 & 36.331 \\
Inverse Jacobian & 0 & -0.02752 & -0.06725 & -0.05003 & -0.04980 & -0.04045 & 131.231 \\
$\delta_{R-J}^{i}$ & & $0.055033 \%$ & $0.009077 \%$ & $0.551593 \%$ & $1.444987 \%$ & $0.729403 \%$ & \\
\hline
\end{tabular}

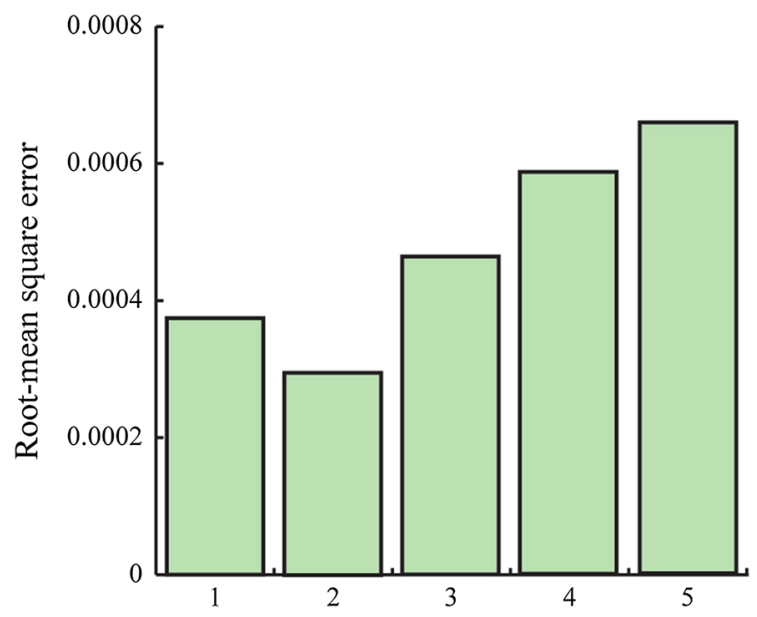

Fig. 2 Root-mean square error of five kinds of disturbance

1) Weight coefficient to rapidity

Using the model, we take different weight coefficients to the rapidity, and then obtain the optimal distribution of all the thermal units by means of improved genetic algorithm (Fig. 3). Figure 4 describes the trend of the regulation time and economical cost with the increase of weight coefficient to the rapidity.

We can see from Fig. 3 the ramping capacity of Unit 5 is superior to the other units, while the economy of Units 2 and 13 are superior to the others. When the weight coefficient of rapidity increases, the simulation results show that the unit with faster ramping capacity will obtain more allocation. Due to the increase of the rapidity weight, the regulation time of the system is reduced at the expense of the efficiency.

In Fig. 3, the optimal distribution curves of Unit 1 and Unit 11 nearly overlap as the weight coefficient to rapidity increases. The reason of the phenomenon above is that Unit 1 and Unit 11 have the same ramping capacity. As the weight coefficient $\alpha_{s}$ increases to a certain high degree, the objective of rapidity ought to be dominant. Thus, the units with same ramping capacity will get similar distributions.

In Fig. 4, the regulation time decreases significantly when weight coefficient to rapidity $\alpha_{\mathrm{s}}$ increases from 0 to 0.2 . With the increase of $\alpha_{\mathrm{s}}$ from 0.2 to 1.0 , the ascent of the rapidity is not obvious in contrast with a marked increase in the generation cost. We can illustrate that we will get a better optimization result when $\alpha_{\mathrm{s}}$ takes the value in the vicinity of 0.2 in our example.

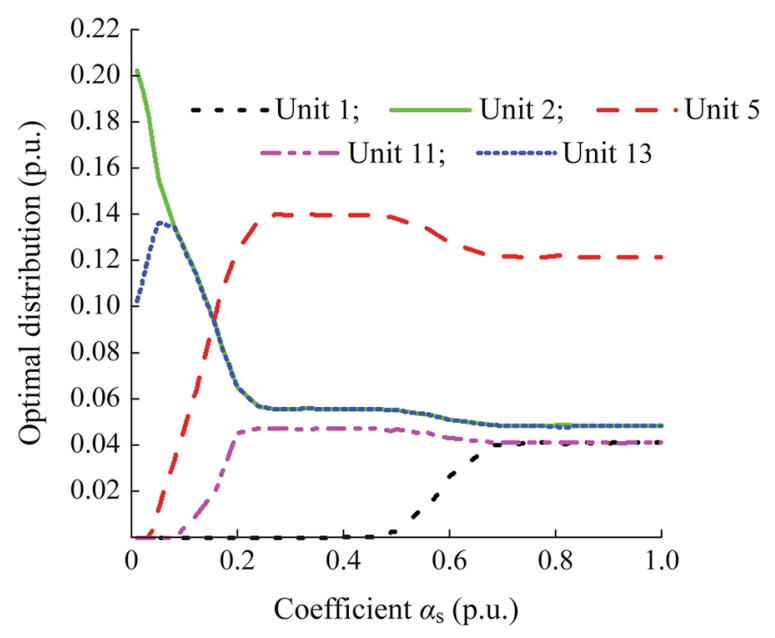

Fig. 3 Impact of $\alpha_{\mathrm{s}}$ on optimal dispatch

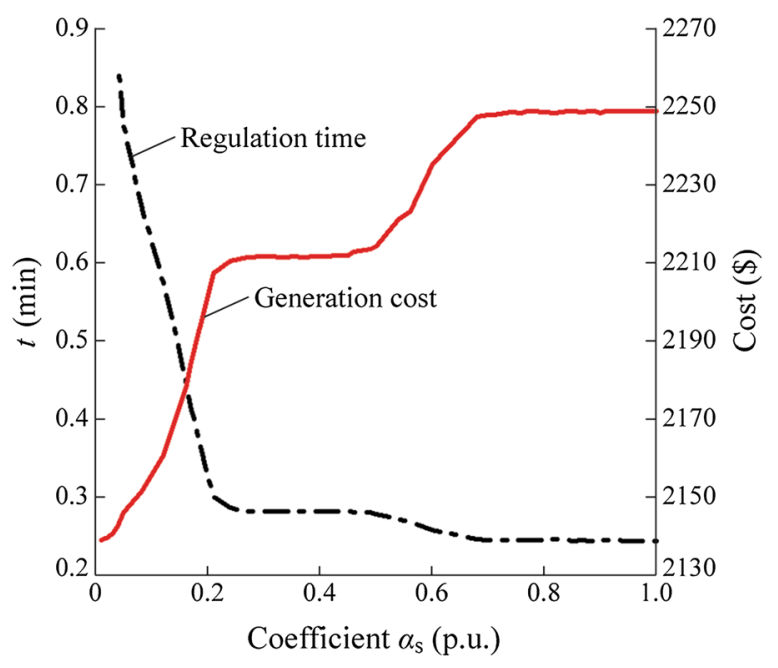

Fig. 4 Impact of $\alpha_{\mathrm{s}}$ on objective function

2) Loss sensitivity

In this section, we analyze the impact of the loss sensitivity on AGC dispatch. We assume the transmission loss as a constant ( 0.03 and 0.06 , respectively), thus obtaining the corresponding optimal distribution in Table 2, wherein the weight is taken as 0.2. As a constast, the optimal distribution when loss sensitivity taken into account is shown in Table 2.

We can see from the Table 2, it will result in different optimal allocations when we ignore the correction of 
Table 2 Impact of transmission loss correction on optimal dispatch

\begin{tabular}{llllllll}
\hline Situation & Unit 1 & Unit 2 & Unit 5 & Unit 11 & Unit 13 & Objective function & $\begin{array}{l}\text { Generation } \\
\text { cost }(\$)\end{array}$ \\
\hline Constant loss (A-0.03) & 0 & 0.1784 & 0.0610 & 0.0091 & 0.0713 & 0.188 & 2168.3 \\
time (min)
\end{tabular}

Table 3 Impact of the location of faster-ramping unit on optimal dispatch

\begin{tabular}{lllllllll}
\hline Unit & Unit A & Unit B & $\begin{array}{l}\text { Unit C } \\
\text { (faster-ramping) }\end{array}$ & Unit D & Unit E & $\begin{array}{l}\text { Objective } \\
\text { function }\end{array}$ & $\begin{array}{c}\text { Generation } \\
\text { cost }(\$)\end{array}$ & $\begin{array}{c}\text { Regulation } \\
\text { time (min) }\end{array}$ \\
\hline 1 & 0.0416 & 0.0497 & 0.1239 & 0.0421 & 0.0496 & 0.1103 & 2255.9 & 0.248 \\
2 & 0.0391 & 0.0492 & 0.1231 & 0.0419 & 0.0492 & 0.1099 & 2248.1 & 0.247 \\
5 & 0.0405 & 0.0487 & 0.1219 & 0.0415 & 0.0487 & 0.1049 & 2248.0 & 0.244 \\
11 & 0.0415 & 0.0489 & 0.1223 & 0.0416 & 0.0489 & 0.1054 & 2251.1 & 0.245 \\
13 & 0.0408 & 0.0492 & 0.1231 & 0.0418 & 0.0492 & 0.1088 & 2251.4 & 0.246 \\
\hline
\end{tabular}

transmission loss or not. It is also illustrated that the results between different constants. The data in Table 2 demonstrate the importance of transmission loss correction.

3) Location of faster-ramping unit

We define Unit A as the unit which was placed at Node 1 in Section 4.2, Unit B as the one at Node 2, Unit C as the one at Node 5, Unit D as the one at Node 11 and Unit $\mathrm{E}$ as the one at Node 13. We exchange the position of Unit C which has faster-ramping capacity with other units respectively, getting the corresponding optimal dispatch of each unit and the objective of rapidity and economy as shown in Table 3, where the rapidity weight is taken as 0.8 . The data in the first column means the node number to which Unit $\mathrm{C}$ is changed, while the optimal generation dispatch is shown in Column 2-5.

The results in Table 3 indicate that the location of fasterramping unit influences the optimal dispatch as well. When the faster-ramping unit is located at Node 5, the system gets the smallest optimization goal, while in Node 1, the optimization goal reaches largest. It can be explained through the distances between faster-ramping unit and Node 8 where wind units are placed. When we take the weight to rapidity as 0.8 , it means the output of faster-ramping unit will account for a large proportion of the total output. In other words, the transmission loss that the faster-ramping unit generates will largely affect the total active power output to adjust. Due to the electrical distance between Node 5 and 8 is the shortest, which means corresponding transmission loss is smallest when the faster-ramping unit is placed at Node 5, the total output is smallest. Similarly, when the fast-ramping unit is placed at Bus 1, the total output reaches the largest. We can conclude that an improved dispatch will be achieved under the condition and that the faster-ramping unit is placed near the wind units.

\section{Conclusion}

In this paper, a fast online approach based on the RLS method is proposed to calculate loss sensitivity using real-time data by PMUs, it helps to set up the power balance constraint. This sensitivity is obtained through identifications based on the measurements in the real power system, instead of simulations based on offline models and parameters, thus it can reflect the real relationships between the wind farms and thermal generators. Also, an improved multi-objective optimization model of dispatch is proposed, which establishes a connection between the thermal units with fast ramping capacity and the wind farms with rapid fluctuations more directly and reasonably.

The simulation is carried out in the IEEE 30-bus system to show the effectiveness of the method. The loss sensitivity is compared with the B-coefficient and inverse Jacobian transpose methods and the results validate its feasibility. AGC units dispatch model is solved with GA. Effects of the weight coefficient on rapidity and the location of fasterramping unit are analyzed. The simulation results verify the validity and the feasibility of the optimization model.

Acknowledgement This work is supported in part by Major State Basic Research Development Program of China (No. 2012CB215206) and National Natural Science Foundation of China (No. 51107061).

Open Access This article is distributed under the terms of the Creative Commons Attribution 4.0 International License (http:// creativecommons.org/licenses/by/4.0/), which permits unrestricted use, distribution, and reproduction in any medium, provided you give appropriate credit to the original author(s) and the source, provide a link to the Creative Commons license, and indicate if changes were made.

\section{Appendix}

The parameters of five thermal units are shown in Table 4. 
Table 4 Parameters of thermal units

\begin{tabular}{llllllllll}
\hline Unit & Power output (p.u.) & $\begin{array}{l}\text { Minimum } \\
\text { capacity } \\
\text { (p.u.) }\end{array}$ & $\begin{array}{l}\text { Maximum } \\
\text { capacity } \\
\text { (p.u.) }\end{array}$ & $a_{j}(\$)$ & $b_{j}(\$ /$ p.u.) & $c_{j}\left(\$ /(\text { p.u. })^{2}\right)$ & $e_{j}(\$)$ & $f_{j}(\mathrm{rad} /$ p.u. $)$ & $V_{j}(\mathrm{p}$. u./min $)$ \\
\hline 1 & 1.2132 & 0.50 & 2.0 & 786.799 & 38.5397 & 0.1524 & 450 & 0.041 \\
2 & 0.2700 & 0.25 & 1.0 & 945.633 & 46.1591 & 0.1058 & 300 & 0.036 & 0.17 \\
5 & 0.5000 & 0.15 & 1.0 & 1049.99 & 40.3965 & 0.0280 & 320 & 0.028 & 0.2 \\
11 & 0.3500 & 0.10 & 0.6 & 1658.56 & 36.3278 & 0.0211 & 280 & 0.063 & 0.17 \\
13 & 0.2500 & 0.10 & 0.6 & 1356.65 & 38.2704 & 0.0179 & 310 & 0.048 \\
\hline
\end{tabular}

\section{References}

[1] Papalexopoulos AD, Andrianesis PE (2014) Performance-based pricing of frequency regulation in electricity markets. IEEE Trans Power Syst 29(1):441-449

[2] Liu XP, Tian L, Wang Q (2014) A control method of rapid load change for heat supply units compensating wind power disturbance. Autom Electr Power Syst 38(6):26-32. doi:10.7500/ AEPS20130701001 (in Chinese)

[3] Tian YF, Zhao WJ, Tian L (2014) Optimum design of fast response for thermal power unite load instructions. Autom Instrum 1:38-42 (in Chinese)

[4] Ren Y, Zhang XQ (2010) Research of unit commitment in power system based on improved dynamic programming method. Tech Autom Appl 29(5):6-8 (in Chinese)

[5] Li WD, Wu HB, Wu YG et al (2003) Application of genetic algorithm to AGC service dispatch in a deregulated power system. Autom Electr Power Syst 27(15):20-24 (in Chinese)

[6] Dudek G (2004) Unit commitment by genetic algorithm with specialized search operators. Electr Power Syst Res 72(3):299-308

[7] Jaini A, Musirin I, Aminudin N et al (2010) Particle swarm optimization (PSO) technique in economic power dispatch problems. In: Proceedings of the 4th international power engineering and optimization conference (PEOCO'10), Shah Alam, Malaysia, 23-24 June 2010, pp 308-312

[8] Zhao B, Guo CX, Bai BR et al (2006) An improved particle swarm optimization algorithm for unit commitment. Int J Electr Power Energy Syst 28(7):482-490

[9] Wang YJ, Fang DZ (2007) Research on AGC unit dispatch based on particle swarm optimization algorithm. Relay 35(17):58-61 (in Chinese)

[10] Wang JG, Cong C, Han N et al (2013) Research of improved immune algorithm for economic AGC unit dispatch. Power Syst Prot Control 41(18):32-36 (in Chinese)

[11] Kirchmayer LK (1958) Economic operation of power systems. Wiley, New York

[12] Dommel HW, Tinney WF (1968) Optimal power flow solutions. IEEE Trans Power Appar Syst 87(10):1866-1876

[13] Conejo AJ, Galiana FD, Kockar I et al (2001) Z-bus loss allocation. IEEE Trans Power Syst 16(1):105-110
[14] Zhang XH, Zhao JQ, Chen XY (2011) Multi-objective unit commitment modeling and optimization for energy-saving and emission reduction in wind power integrated system. Power Syst Prot Control 39(17):33-39 (in Chinese)

[15] Sun YZ, Wu J, Li GJ et al (2009) Dynamic economic dispatch considering wind power penetration based on wind speed forecasting and stochastic programming. In: Proceedings of the CSEE

Jingyi ZHANG received the B.E. degree from Tianjin University in 2013. From 2013, she started to pursue her Ph.D. degree in Tsinghua University. Her current research focuses on the optimal dispatch of AGC units.

Chao LU received the B.E. and Ph.D. degrees from Tsinghua University in 1999 and 2005, respectively. Now, he works as an associate professor in the Department of Electrical Engineering at Tsinghua University. His research interests include power system stability and control.

Jie SONG received the B.S. degree in applied mathematics from Peking University in 2004, and the Ph.D. degrees in industrial engineering from Tsinghua University in 2010. She is an Assistant Professor with the Department of Industrial Engineering and Management, Peking University. Her research interests are simulation optimization, stochastic modeling.

Junbo ZHANG was born in Guizhou, China, on 16 March 1986. He received his B.Eng. and Ph.D. (with First Class Honors) from Tsinghua University in 2008 and 2013, respectively, and is now a postdoc fellow at the same University. He studied at The Hong Kong Polytechnic University from 2009 to 2011 under a collaborative program between Tsinghua University and The Hong Kong Polytechnic University. His research areas include wide-area based power system analysis and control, signal processing and system identification in power system. 